\title{
Neuropsychiatric symptoms in Vascular Cognitive Impairment
}

\author{
A systematic review
}

\author{
Chan Tiel ${ }^{1}$, Felipe Kenji Sudo², Gillberto Sousa Alves ${ }^{2,3}$, Letice Ericeira-Valente², \\ Denise Madeira Moreira" ${ }^{4}$, Jerson Laks ${ }^{2,5}$, Eliasz Engelhardt ${ }^{1}$
}

\begin{abstract}
Neuropsychiatric symptoms or Behavioral and Psychological Symptoms of Dementia (BPSD) are common and invariably appear at some point during the course of the disease, mediated both by cerebrovascular disease and neurodegenerative processes. Few studies have compared the profiles of BPSD in Vascular Cognitive Impairment (VCI) of different subtypes (subcortical or cortical) and clinical stages (Vascular Cognitive Impairment No Dementia [VaCIND] and Vascular Dementia [VaD]]. Objective: To review the BPSD associated with different subtypes and stages of VCl using the Neuropsychiatric Inventory (NPI). Methods: Medline, Scielo and Lilacs databases were searched for the period January 2000 to December 2014, with the key words: "BPSD AND Vascular Dementia, "NPI AND Vascular Dementia" and "NPI AND VCI. Qualitative analysis was performed on studies evaluating BPSD in VCl, using the Neuropsychiatric Inventory (NPI). Results: A total of 82 studies were retrieved of which 13 were eligible and thus included. Among the articles selected, 4 compared BPSD in Subcortical Vascular Dementia (SVaD) versus Cortical-Subcortical Vascular Dementia (CSVaD), 3 involved comparisons between SVaD and VaCIND, 1 study analyzed differences between CSVaD and VaCIND, while 5 studies assessed BPSD in CSVaD. Subcortical and Cortical-Subcortical VaD were associated predominantly with Apathy and Depression. VaCIND may present fewer behavioral symptoms than VaD. Conclusion: The profile of BPSD differs for different stages of $\mathrm{VCl}$. Determining the most prevalent BPSD in VCl subtypes might be helpful for improving early diagnosis and management of these symptoms.
\end{abstract}

Key words: neuropsychiatric symptoms, BPSD, NPI, Vascular Cognitive Impairment, Vascular Dementia, Alzheimer's disease.

\section{SINTOMAS NEUROPSIQUIÁTRICOS NO COMPROMETIMENTO COGNITIVO VASCULAR: REVISÃO SISTEMÁTICA}

RESUMO. Sintomas Neuropsiquiátricos ou Sintomas de Comportamento e Psicológicos da Demência (SCPD) são comuns e aparecem em algum ponto no curso da enfermidade, mediado por doenças cerebrovasculares e processos neurodegenerativos. Poucos estudos compararam os perfis dos SCPD no Comprometimento Cognitivo Vascular (CCV) de diferentes subtipos (subcortical ou córtico-subcortical) e diferentes estágios clínicos (Comprometimento Cognitivo Vascular Não Demência [CCV-ND] e Demência Vascular [DV]). Objetivo: Revisar os SCPD associados aos diferentes subtipos e estágios do CCV, com o Inventário Neuropsiquiátrico (INP). Métodos: Medline, Scielo e Lilacs foram pesquisados de janeiro de 2.000 até dezembro de 2.014, com as palavras chaves: "BPSD AND Vascular Dementia", "NPI AND Vascular Dementia" e "NPI AND VCI". Uma análise quantitativa foi feita nos estudos que avaliaram sintomas comportamentais no CCV através do INP. Resultados: Um total de 82 estudos foram identificados e 13 estudos foram considerados elegíveis e incluídos. Destes artigos, 4 compararam SCPD entre DV Córtico-Subcortical (DVCS) e DV Subcortical (DVS), 3 artigos compararam DVS e CCVND, 1 artigo analisou diferenças entre DVCS e CCVND, e 5 estudos avaliaram os SCPD em DVCS. Tanto a DVCS quanto a DVS associaram-se principalmente a Apatia e Depressão. 0 CCVND pode apresentar menos sintomas comportamentais que a DV. Conclusão: 0 perfil dos SCPD difere entre diferentes estágios do CCV. 0 entendimento dos SCPD mais prevalentes nos subtipos do CCV poderia ser útil para facilitar 0 diagnóstico precoce e o manejo desses sintomas.

Palavras-chave: sintomas neuropsiquiátricos, SCPD, INP, Comprometimento Cognitivo Vascular, Demência Vascular, doença de Alzheimer.

\footnotetext{
The study was conducted at the Institute of Psychiatry and Institute of Neurology - UFRJ, Rio de Janeiro RJ - Brazil.

'Instituto de Neurologia Deolindo Couto, Setor de Neurologia Cognitiva e do Comportamento-INDC-CDA/PUB, UFRJ, Rio de Janeiro RJ, Brazil. 2Instituto de Psiquiatria, Universidade Federal do Rio de Janeiro (UFRJ), Rio de Janeiro RJ, Brazil. ${ }^{3}$ Departamento de Medicina Clínica, Universidade Federal do Ceará, Fortaleza CE, Brazil. " 4 Serviço de Radiologia, Instituto de Neurologia Deolindo Couto (UFRJ); Hospital Pró-Cardíaco, Rio de Janeiro RJ, Brazil. 5Universidade do Estado do Rio de
} Janeiro, Rio de Janeiro RJ, Brazil.

Chan Tiel. Rua General Goes Monteiro, 184/904 - 22290-080 Rio de Janeiro RJ - Brazil. E-mail: chantiel10@hotmail.com

Disclosure: The authors report no conflits of interest.

Received April 05,2015. Accepted in final form June 20, 2015. 


\section{INTRODUCTION}

$\mathrm{V}^{2}$ ascular Dementia (VaD) is considered the second-most-common type of dementing illness, accounting for a significant proportion of total dementia cases. ${ }^{1,2}$ Vascular Cognitive Impairment (VCI) is a broader dimensional term, ${ }^{3}$ encompassing subjects with mild cognitive impairment and no incapacity on activities of daily living, referred to as Vascular Cognitive Impairment No Dementia (VaCIND), as well as conditions associated with significant cognitive impairment and decline in functional status (VaD), ${ }^{4,5}$ Clinical features can vary considerably depending on the extension and location of vascular lesions. Moreover, VCI may be associated with large-vessel disease, which often leads to extensive cortical-subcortical damage, or with insidious occlusion/semi-occlusion of small penetrating arterioles, resulting in subcortical vascular disease. ${ }^{6,7}$

Comparisons among signs and symptoms of different subtypes of VCI may help understand how different mechanisms of brain damage can produce convergent or divergent clinical features, which in turn may lead to a better comprehension of the pathophysiology of the disorder. Among these manifestations, Neuropsychiatric Symptoms are of great importance, considering that they almost invariably appear at some point of the natural course of VCI in addition to cognitive impairment..$^{8-12}$ Also referred as Behavioral and Psychological Symptoms of Dementia (BPSD), ${ }^{13}$ these features are frequently associated with growing levels of distress both in persons with dementia and in their caregivers, as well as with higher risk for adverse outcomes and increased use of health care resources. ${ }^{14}$ Clinically, BPSD have a major impact on the patient's functional and cognitive status. ${ }^{15}$

Despite considerable advances in the detection of brain vascular-related syndromes in recent years, the association between vascular lesions and both cognitive symptoms and BPSD in VCI remains controversial. For instance, data in the literature suggests that BPSD may occur in VCI, regardless of the development of dementia. ${ }^{16,17}$ BPSD may also appear at any stage, induced by cerebrovascular lesions disrupting the cortical-subcortical circuits between prefrontal cortex connections to limbic nuclei of the basal ganglia and thalamus, and other limbic system structures. ${ }^{18-20}$ This suggests that better characterization of vascular-related BPSD, and the underlying mechanisms of brain injury associated with these features, is still needed so as to allow the adoption of effective evidence-based prophylactic and therapeutic measures.

Among methods for assessing BPSD, the Neuropsychiatric Inventory (NPI) ${ }^{21}$ is a valid and reliable instru- ment, originally developed to assess 10 neuropsychiatric disturbances commonly seen in dementia. Subsequently, it has been modified to evaluate 12 disturbances including agitation, aberrant motor behavior, anxiety, elation, irritability, depression, apathy, disinhibition, delusions, hallucinations, sleep and appetite changes..$^{22}$ Currently, the NPI is the most frequently used instrument for evaluating these symptoms in research studies and clinical practice. It is a semistructured interview which rates the frequency and severity of BPSD for the month preceding assessment. The NPI has been translated and validated to Brazilian Portuguese. ${ }^{23}$

To date, few studies have compared neuropsychiatric disturbances among VCI subgroups (cortical or subcortical, "pure" or mixed etiology, VaD or VaCIND). Thus, the aim of the present review was to examine BPSD profiles in VCI subgroups, primarily addressing two issues: [1] which are the most prevalent BPSD in VCI, and [2] whether different clinical presentations of $\mathrm{VCI}$ present different BPSD profiles. It can be hypothesized that the BPSD in VCI have a heterogeneous pattern, depending on the clinical stage and mechanism of brain lesion.

\section{METHODS}

Search strategy and criteria. To identify articles, a systematic search was performed in PUBMED/MedLine, Scielo and Lilacs databases for English-language articles published between January 2000 and December 2014, followed by a manual search of the reference lists of relevant articles retrieved. The results of three searches, which employed the following key words: "BPSD AND Vascular Dementia", "NPI AND Vascular Dementia" and "NPI AND VCI" were combined.

Studies were selected for inclusion in the present systematic review if they met all of the following criteria: [a] studies that assessed patients diagnosed with $\mathrm{VaD}$ according to the $4^{\text {th }}$ edition of the Diagnostic and Statistical Manual of Mental Disorders (DSM-IV) ${ }^{24}$ criteria or the NINDS-AIREN criteria ${ }^{1}$ and/or studies that assessed Va-CIND subjects, diagnosed according to wellrecognized criteria; ${ }^{24,26}[\mathrm{~b}]$ studies that assessed BPSD in VCI; [c] studies that evaluated BPSD using the NPI; [d] studies that presented data on the prevalence and/or incidence rates of BPSD. Studies involving Alzheimer's disease (AD) or mixed dementia (MD) subjects were included if within a comparison group for $\mathrm{VCI}$ subjects.

The following exclusion criteria were used: [a] articles with $\mathrm{AD}$ samples only; [b] studies that assessed dementia other than $\mathrm{AD}$ or $\mathrm{VaD}$; $[c]$ clinical trials with medications; [d] reviews, posters, case reports, essays or letters. 


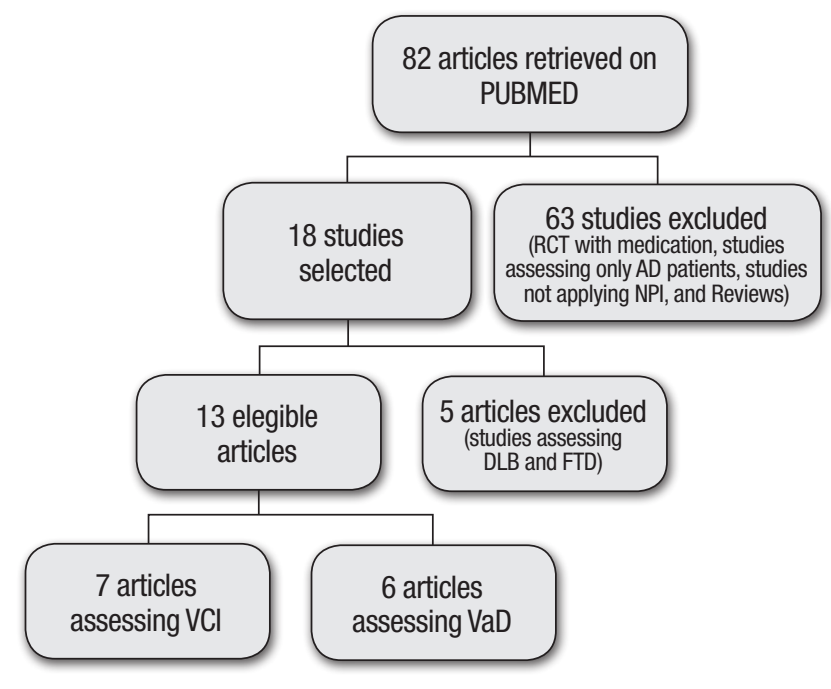

RCT: Randomized clinical trial; AD: Alzheimer's Disease; NPI: Neuropsychiatric Inventory; DLB: Dementia with Lewy Bodies; FTD: Frontotemporal dementia; VCl: Vascular Cognitive Impairment; VaD: Vascular Dementia.

Figure 1. Flowchart of search strategy and article selection.

Data extraction. Data from those studies meeting the inclusion criteria were extracted by one of the authors (CT) and reviewed by other researchers from the group (JL, FKS). In cases of disagreement among the two, discussion included the whole group to reach a consensus. Information about the clinical diagnosis, sample size, assessment instrument for BPSD, and the prevalence of BPSD categories were drawn from the selected studies.
Statistical analysis. Data were analyzed using the IBM Statistical Package for Social Sciences version 20 for Windows. Initially, the Shapiro-Wilk test was performed to verify the normality of data (prevalence of BPSD). Since significance exceeded .05 for all variables, One-Way ANOVA was performed to compare statistically significant differences between BPSD prevalence in the SVaD (Subcortical Vascular Dementia), VaCIND (Vascular CIND) and CSVaD (Cortical-Subcortical Vascular Dementia) groups. Tukey's Post-hoc test was performed to identify groups with significantly different prevalences of BPSD. The level of significance was set at .05.

\section{RESULTS}

Of the total 82 articles initially retrieved, 13 fulfilled the inclusion criteria and were included in this review. No studies were identified on the Lilacs and Scielo databases using the previously described search strategy. Figure 1 shows the stages of selection of papers included in this article.

The number of participants in the studies ranged from 51 to 938 patients. Diagnostic groups included different stages of VCI (VaCIND and $\mathrm{VaD}$ ), etiologies (VCI, AD and MD) and subtypes of $\mathrm{VaD}$ (cortical and cortical-subcortical). Operational criteria for VCI were the DSM-IV, ${ }^{24}$ NINDS-AIREN, ${ }^{1}$ Hachinski's Ischemic Score,${ }^{27}$ Clinical Dementia Rating, ${ }^{28}$ Fazekas scale, ${ }^{29}$ and the Canadian Study of Health and Ageing criteria for VaCIND. ${ }^{30}$ These data are summarized in Table 1.

Table 1. Characteristics of samples, diagnostic groups and operational criteria in the studies selected.

\begin{tabular}{|c|c|c|c|c|}
\hline Year & Diagnosis & $\mathbf{n}$ & Diagnostic criteria & References \\
\hline 2007 & $\mathrm{~V}-\mathrm{CIND}+\mathrm{VaD}+\mathrm{MD}(\mathrm{AD}+\mathrm{VaD})$ & 157 & DSM-IV, CDR, HIS & Chiu et al. ${ }^{17}$ \\
\hline 2010 & $\mathrm{VaD}$ & 484 & DSM-IV, NINDS-AIREN & Staekenborg et al. ${ }^{42}$ \\
\hline 2012 & SVD + V-CIND & 73 & DSM-IV, Fazekas, CDR & Chin et al. ${ }^{40}$ \\
\hline 2013 & SVD + V-CIND & 127 & DSM-IV, Fazekas, CDR & Kim et al. ${ }^{43}$ \\
\hline 2013 & VaD + V-CIND & 60 & NINDS-AIREN, DSM-IV, HiS, CDR & Gupta et al. ${ }^{31}$ \\
\hline 2013 & $\mathrm{VaD}$ & 51 & NINDS-AIREN,DSM-IV & Pan et al. ${ }^{44}$ \\
\hline 2014 & VaD + V-CIND & 60 & NINDS-AIREN, CSHA & Gupta et al. ${ }^{45}$ \\
\hline 2000 & $A D+V a D$ & 329 & DSM-IV, NINDS-AIREN, CDR & 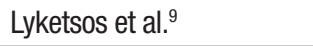 \\
\hline 2005 & $A D+$ VaD (cortical + subcortical) & 536 & DSM-IV, NINCDS-ADRDA, CDR & Fuh et al. ${ }^{35}$ \\
\hline 2009 & $A D+V a D$ & 154 & DSM-IV, CDR, NINCDS-AIREN & Hsieh et al. ${ }^{15}$ \\
\hline 2008 & $A D+$ VaD (cortical + subcortical) & 65 & DSM-IV, NINCDS-ADRDA, HIS & Fernández-Martínez et al. ${ }^{32}$ \\
\hline 2012 & $A D+V a D$ & 302 & DSM-IV, NINCDS-ADRDA, NINDS-AIREN & D’onófrio et al. ${ }^{46}$ \\
\hline 2014 & $A D+V a D$ (cortical + subcortical) & 100 & DSM-IV, NINDS-AIREN & Bandyopadhyay et al..$^{33}$ \\
\hline
\end{tabular}

AD: Alzheimer Dementia; VaD: Vascular Dementia; MD: Mixed Dementia; SVD: Subcortical Vascular Dementia; VCIND: Vascular cognitive Impairment-No Dementia; DSM-IV: Diagnostic and Statistical Manual of Mental Disorders $4^{\text {th }}$ edition; CDR: Clinical Dementia Rating; HIS: Hachinski ischaemic Score; Fazekas: Fazekas scale; CSHA: Canadian Study of Health and Ageing; NINDS-AIREN: National Institute of Neurological Disorders and Stroke and Association - Internationale pour la Recherché et IÉnseignement en Neurosciences; NINCDS-ADRDA: National Institute of Neurological and Communicative Disorders and Stroke and the Alzheimer's Disease and Related Disorders Association. 
BPSD were identified in all diagnostic groups at a high prevalence: 83.8 [96.4\%] in SVaD, 59.7 [100\%] in CSVaD and 47.5 [89\%] in VaCIND. Apathy and Depression were the most frequent BPSD, followed by Irritability, Anxiety and Agitation. In another study, a higher prevalence of Euphoria was identified in VaCIND than in $\mathrm{VaD}$ (7.14\% vs 3.13\%). ${ }^{31}$ Agitation/Aggression symptoms appeared to be equally prevalent in CSVaD (21.4 [62.9\%]) and in SVaD (22.7 [47.62\%]). Also, patients with SVaD presented comparable Aberrant Motor Behavior (up to $38.10 \%$ ) and Hallucinations (up to $28 \%$ ) as patients with CSVaD (5-61.5\% and 1-30.8\%, respectively). Table 2 depicts the prevalences of BPSD and the NPI scores in the studies.

On comparison of mean prevalence of BPSD among subtypes of VCI, Apathy and Agitation were found to differ significantly in the assessed groups. Agitation was significantly more prevalent in CSVaD than in VaCIND $(\mathrm{p}=0.036)$, whereas the VaCIND group showed significantly less Apathy than both SVaD and CSVaD groups $(p=0.025)$. Table 3 shows these results.

\section{DISCUSSION}

The present study reviewed the characteristics of BPSD in VCI of different subtypes and clinical stages. The articles retrieved in this search showed that at least half of those individuals with VCI presented BPSD. Moreover, comparison between BPSD in SVaD and CSVaD revealed no differences in prevalence and profile of BPSD domains. Therefore, both subtypes of $\mathrm{VaD}$ are likely to present Depression and Apathy during the course of the disease. Other symptoms varied widely in prevalence among groups. As expected, VaCIND subjects had a lower prevalence of BPSD than $\mathrm{VaD}$ patients, a finding consistent with larger areas of spared brain tissue. Studies showed higher frequency of Delusions, Aberrant Motor Behavior and Sleep disorders in $\mathrm{AD}$ than in VaD subjects. ${ }^{9,15,32,35}$

Although $\mathrm{AD}$ was not part of the present search, available data on the differences between BPSD in vascular and neurodegenerative cognitive impairments were also examined. Studies that assessed AD also showed high prevalence of BPSD (up to 53.3\%). More than half of the patients had at least one symptom, both in $\mathrm{VaD}$ and $\mathrm{AD}$ ( $\mathrm{AD} 53.3 \%$ vs $\mathrm{VaD} 59.7 \%$ ). ${ }^{9}$ In three studies, almost all patients with $\mathrm{AD}$ or $\mathrm{VaD}$ had some type of $\mathrm{BPSD}(\mathrm{AD}=100 \%$ vs $\mathrm{VaD}=100 \% ; \mathrm{AD}=94.6 \%$ vs $\mathrm{VaD}=96.4 \%)^{15,29,30}$ Comparisons of BPSD prevalence in $\mathrm{VaD}$ and $\mathrm{AD}$ showed heterogeneous results. Depression and Apathy were more prevalent in $\mathrm{VaD}$, whereas prevalence of the other symptoms varied widely across the studies. In another study, the prevalence for Depression was also higher in VCI (VCI $=30 \%$ vs $\mathrm{AD}=15 \%){ }^{31}$ Several studies reported a higher prevalence of specific BPSD domains in $\mathrm{AD}$ than in $\mathrm{VaD}$, as follows: Delusions ( $\mathrm{AD}=22.4 \%$ vs $\mathrm{VaD}=8.1 \%),{ }^{9}$ Aberrant Motor Behavior $(\mathrm{AD}=24.3 \%$ vs $\mathrm{VaD}=7.1 \%),{ }^{32}$ Nocturnal Behavior/Sleep ( $\mathrm{AD}=96.0 \%$ vs $\mathrm{VaD}=73.1 \% / \mathrm{AD}=35.1 \%$ vs $\mathrm{VaD}=3.6 \%)^{15,32}$ These results indicate different patterns of behavioral symptoms between $\mathrm{VCI}$ and $\mathrm{AD}$. However, since $\mathrm{AD}$ was not part of the search strategy for this review, further studies evaluating BPSD differences between VCI and $\mathrm{AD}$ are needed to allow more robust conclusions.

A number of issues in assessing BPSD may account for the disparity in results among studies. Neuropsychiatric Symptoms in dementia tend to fluctuate over time. Therefore, estimating prevalence of BPSD using a crosssectional approach may not be appropriate. Moreover, since assessment of individual BPSD domains resulted in great variability across studies, identifying clusters of symptoms with similar underlying neurobiological correlates may allow better characterization of the behavioral profiles of VCI subtypes. In this regard, Frisoni et al. $(1999)^{36}$ described a three-factor model of BPSD in AD: Mood (indicated by Anxiety, Apathy and Depression), Psychotic (Irritability/Lability, Delusions, Hallucinations and Agitation/Aggression), and Frontal Symptoms Domain (Euphoria and Disinhibition). Aalten et al. $(2008)^{37}$ identified four neuropsychiatric subsyndromes: hyperactivity, psychosis, affective syndrome and apathy. Truzzi et al. (2013) ${ }^{38}$ compared BPSD clusters in subjects with dementia evaluated in two different countries: the Brazilian sample showed factors, such as Psychosis (Delusion, Hallucination, Euphoria, and Disinhibition), Mood (Depression, Anxiety, Agitation/Aggression, and Irritability), and Psychomotor (Apathy and Aberrant Motor Behavior), whereas factors drawn from a Norwegian sample were Psychosis (Delusion, Hallucination, and Aberrant Motor Behavior), Mood (Depression, Anxiety, Agitation/Aggression, Apathy and Irritability), and Frontal (Euphoria, Disinhibition, and Irritability).

Applying the cluster-model to the results of this review allows several conclusions to be drawn. Mood and psychomotor symptoms were more prevalent in patients with VCI whereas patients with AD may have more psychotic symptoms. Mood symptoms appeared to be equally prevalent in both $\mathrm{SVaD}$ and $\mathrm{CSVaD}$.

Among the subtypes of VCI, data suggests that Apathy and Agitation were more prevalent in $\mathrm{VaD}$ than in VaCIND. Some differences between SVaD and CSVaD were identified. Agitation significantly differed in CSVaD as compared to VaCIND, but this difference was not 


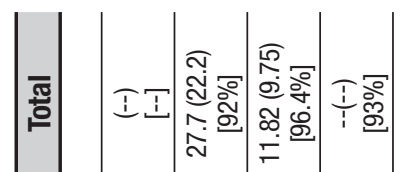

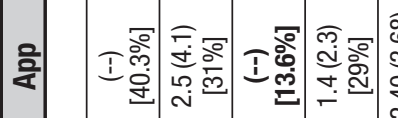

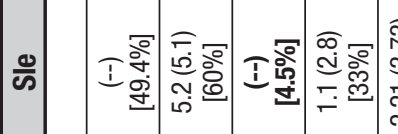

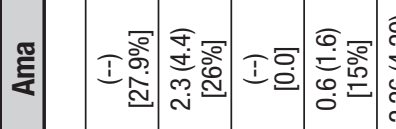

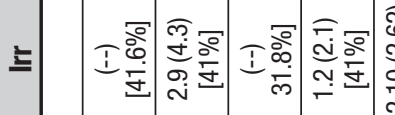

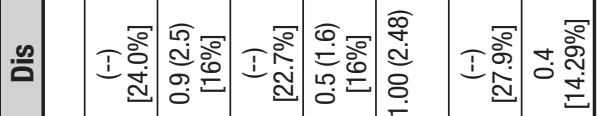

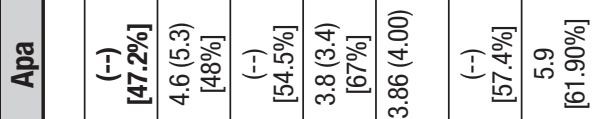

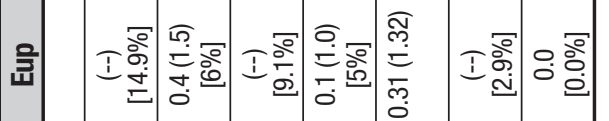

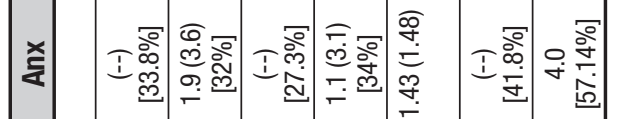

畩

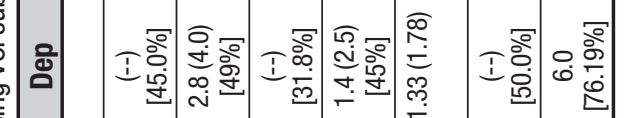

,

:

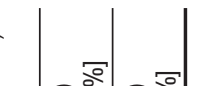
I.

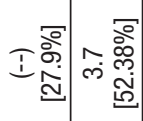

I

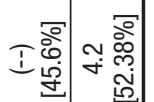

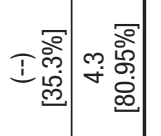

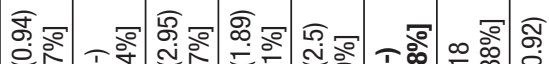

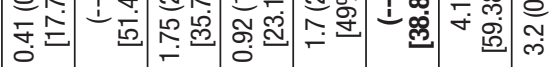

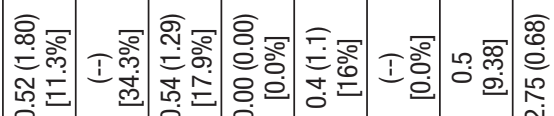

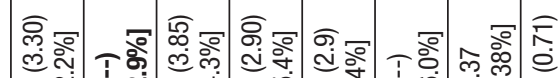
管

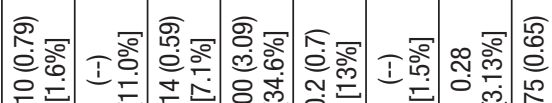

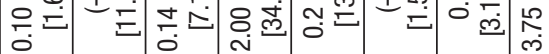

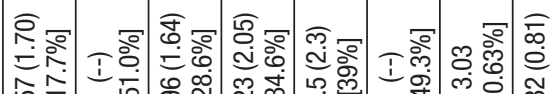
荾

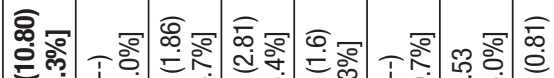

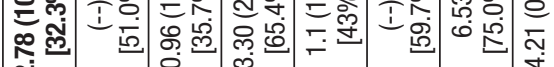

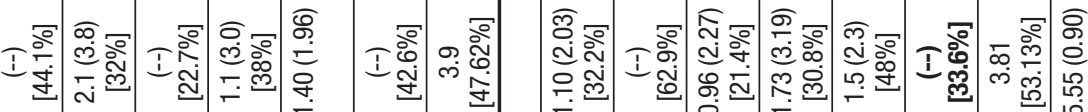

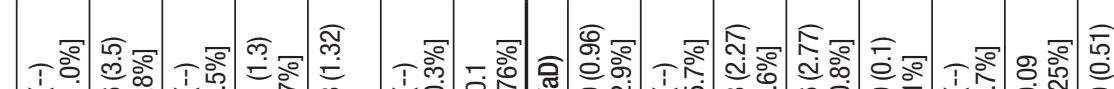
I I.

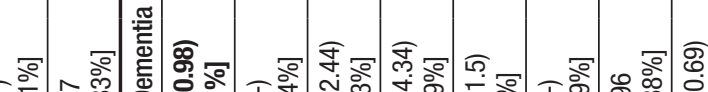

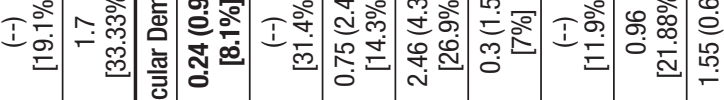

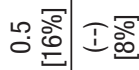

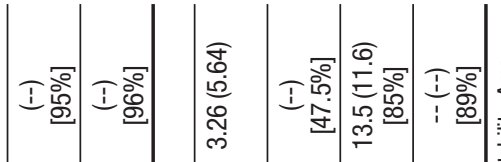
חִ 舀

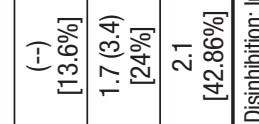

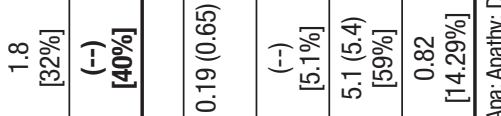

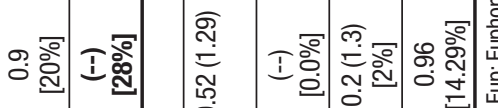

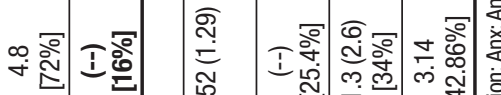

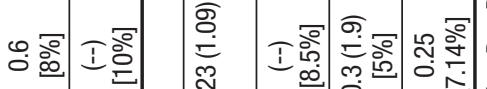
คำ

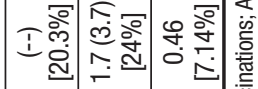
人 m N更造

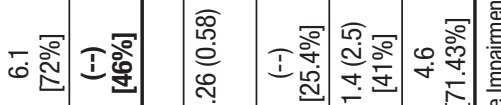

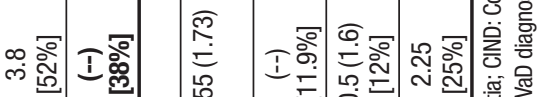

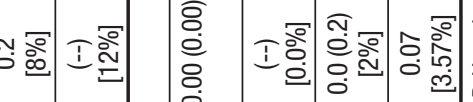
离 흘 率

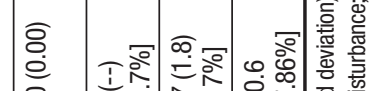

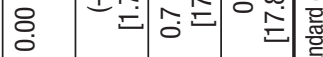
焉 $\frac{8}{\omega}$

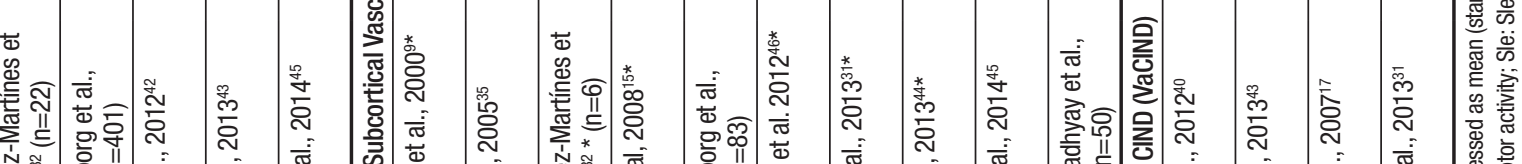

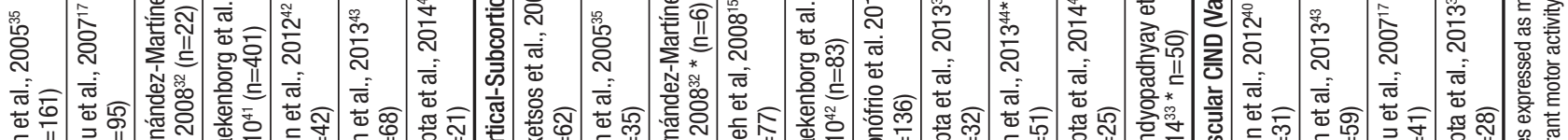

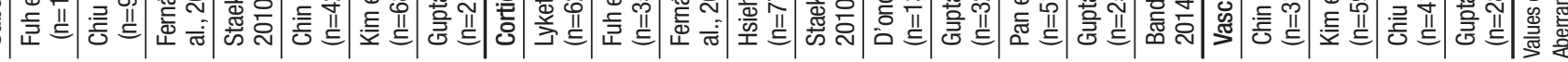


Table 3. Comparison of mean Neuropsychiatric Symptom prevalences among SVaD, CSVaD and VaCIND groups.

\begin{tabular}{|c|c|c|c|c|c|}
\hline Neuropsychiatric symptoms & SVaD $(n=810)$ & CSVaD $(n=557)$ & VaCIND $(n=159)$ & p-value & Post-hoc \\
\hline Delusion & $19.68(10.68)$ & $16.16(8.78)$ & $12.18(9.09)$ & .519 & - \\
\hline Hallucination & $10.79(10.06)$ & $12.21(9.92)$ & $1.85(1.78)$ & .272 & - \\
\hline Agitation & $33.45(14.30)$ & $41.33(13.34)$ & $16.30(7.53)$ & .036 & CSVaD $\neq$ VaCIND \\
\hline Depression & $52.63(15.69)$ & $53.34(15.50)$ & 45.94(23.40) & .798 & - \\
\hline Anxiety & $36.37(10.28)$ & $36.09(10.53)$ & $31.28(10.61)$ & .759 & - \\
\hline Euphoria & $5.41(5.30)$ & $9.99(10.20)$ & $3.04(3.68)$ & .350 & - \\
\hline Apathy & $49.02(19.78)$ & $50.24(16.21)$ & $17.14(8.86)$ & .025 & CSVaD $\neq$ VaCIND; SVaD $\neq$ VaCIND \\
\hline Disinhibition & $17.27(9.11)$ & $10.83(11.11)$ & $6.88(1.76)$ & .254 & - \\
\hline Irritability & $38.23(12.21)$ & $40.34(19.32)$ & $34.08(8.73)$ & .839 & - \\
\hline Motor alterations & $17.60(14.28)$ & $22.47(17.23)$ & $5.43(7.73)$ & 2.73 & - \\
\hline Sleeping alterations & $33,47(21,90)$ & $44.85(22.94)$ & 26.13(28.83) & .447 & - \\
\hline Appetite alterations & 37.98 (20.76) & $40.23(28.20)$ & 26.82(14.83) & .710 & - \\
\hline Total & $92.53(4.55)$ & 88.07 (16.42) & $73.83(22.89)$ & .226 & - \\
\hline
\end{tabular}

BPSD: Behavioral and Psychological Symptoms of Dementia; SVaD: Subcortical Vascular Dementia; CSVaD: Cortical-subcortical Vascular Dementia; VaCIND: Vascular Cognitive Impairment No-Dementia.

found in relation to SVaD. This finding suggests a possible role of cortical function in the pathophysiology of this Neuropsychiatric Symptom. In fact, neurofibrillary tangles involving orbitofrontal cortex bilaterally have been previously associated with agitation among $\mathrm{AD}$ patients. ${ }^{19}$ Furthermore, both SVaD and CSVaD significantly differed from VaCIND in the mean prevalence of Apathy, which might indicate the importance of subcortical changes for the genesis of these symptoms. Apathy may be induced by changes in the neural networks generating and controlling goal-directed actions, ${ }^{39}$ which predominantly involve prefrontal cortex connections to basal ganglia, thalamus and limbic system structures. ${ }^{20}$ Thus, it seems plausible that disruption of the whitematter tracts between frontal cortex and basal ganglia by severe white-matter hyperintensities may result in Apathy. In addition, the severity of vascular load might also contribute to the occurrence of Apathy, as suggested by Chin et al. (2012) ${ }^{40}$ who found that patients with $S V a D$ had higher scores for most individual items of the NPI than patients with SVaMCI, especially Apathy.

The present review has some limitations. Studies were included in which NPI was the only instrument evaluating BPSD. The inclusion of studies using other behavioral measurements, such as the Behave-AD, may have precluded direct comparisons among studies due to differences in the evaluated BPSD items evaluated. However, additional instruments would have provided a more overarching characterization of BPSD profiles in dementia subtypes. Moreover, since the NPI was not designed to assess BPSD in subjects with cognitive impairment below the dementia threshold, behavioral disturbances in VaCIND might have been underestimated. Secondly, some studies did not report the prevalence of each NPI item, which may have affected the results in this review. Finally, scoring on the NPI may be influenced by variables associated with the caregiver, such as burden. A version of the NPI which includes the clinician's impression of the patient's behavioral features (Neuropsychiatric Inventory-Clinician rating scale [NPI-C]) has been proposed and data has suggested that it may reduce bias associated with caregivers' imprecise information. ${ }^{41}$

Given the high prevalence, particularly of mood disorders, it is clear that a rigorous assessment of psychiatric features in VCI should be part of the routine examination of this patient group. Characterization of the behavioral profile of these subjects may allow a better comprehension of the disorder's pathophysiology and enable the development of more effective treatments for these conditions, positively impacting patient quality of life.

Author contribution. Design of the study: Eliasz Engelhardt, Jerson Laks. Analysis of the data: Chan Tiel, Felipe Kenji Sudo, Jerson Laks. Intellectual contribution to the writing of the manuscript: Chan Tiel, Felipe Kenji Sudo, Gilberto Sousa Alves, Letice Ericeira-Valente, Denise Madeira Moreira, Eliasz Engelhardt. Statistics: Felipe Kenji Sudo. Manuscript written by: Chan Tiel, Felipe Kenji Sudo. Interim and final revision: Eliasz Engelhardt, Jerson Laks.

Support. Conselho Nacional de Pesquisa (CNPq) for the support to Jerson Laks, who is a Researcher 2 of this council. 


\section{REFERENCES}

1. Román GC, Tatemichi TK, Erkinjunti T, et al. Vascular Dementia: Diagnostic criteria for research studies. Report of the NINDS-AIREN international workshop. Neurology 1993;43:250-260.

2. Román GC. Vascular dementia may be the most common form of dementia in the elderly. J Neurol Sci 2002;203-204:7-10.

3. Hachinski V. Vascular Dementia: A radical redefinition. Dement Geriatr Cogn Disord 1994;5:130-132.

4. Rockwood K, Wentzel C, Hachinski V, Hogan DB, MacKnight C, McDowell I. Prevalence and outcomes of vascular cognitive impairment. Neurology 2000;54:447-451.

5. Rockwood K. Vascular cognitive impairment and vascular dementia. J Neurol Sci 2002;203-204:23-27

6. Román GC, Erkinjuntti, Wallin A, Pantoni L, Chui HC. Subcortical ischaemic vascular dementia. Lancet Neurol 2002;1:426-436.

7. Levine DA, Langa KM. Vascular cognitive impairment: disease mechanisms and therapeutic implications. Neurotherapeutics 2011;8:361373.

8. O'Brien J. Behavioral symptoms in vascular cognitive impairment and vascular dementia. Int Psychogeriatr 2003;15:133-138.

9. Lyketsos CG, Steinberg M, Tschanz JT, Norton MC, Steffens DC, Breitner JC. Mental and Behavioral Disturbances in Dementia: Findings from the Cache County Study on memory in aging. Am J Psychiatry 2000;157:708-714

10. Aalten P, Vugt ME, Jaspers N, Jolles J, Verhey FR. The course of neuropsychiatric symptoms in dementia. Part 1: findings from the two year longitudinal Maasbed study. Int J Geriatr Psychiatry 2005;20:523-530.

11. Aalten P, Verhey FRJ, Boziki M, et al. Neuropsychiatric syndromes in Dementia. Results from the European Alzheimer Disease Consortium part I. Dement Geriatr Cogn Disord 2007;24:457-463.

12. Caputo M, Monastero R, Mariani E, et al. Neuropsychiatric symptoms in 921 derly subject with dementia: a comparison between vascular and neurodegenerative types. Acta Psychiatr Scand 2008;117:455-464.

13. Finkel SI, Costa e Silva J, Cohen G, Miller S, Sartorius N. Behavioral and psychological signs and symptoms of dementia: a consensus statement on current knowledge and implications for research and treatment. Int Psychogeriatr 1996;8:497-500.

14. Cerejeira J, Lagarto L, Mukaetova-Landinska EB. Behavioral and Psychological symptoms of dementia. Front Neurol 2012;3:1-21.

15. Hsieh CJ, Chang CC, Lin CC. Neuropsychiatric profiles of patients with Alzheimer's disease and Vascular Dementia in Taiwan. Int J Geriatr Psychiatry 2009;24:570-577.

16. Eriksson S. Vascular dementia and Alzheimer's disease: Should we study both within the same study? Int Psychogeriatr 1996;8:443-445.

17. Chiu PY, Liu $\mathrm{CH}$, Tsai $\mathrm{CH}$. Neuropsychiatric manifestations in vascular cognitive impairment patients with and without dementia. Acta Neurol Taiwan 2007;16:86-91.

18. Alexopoulos GS. Depression in the elderly. Lancet 2005;365(9475): 1961-1970.

19. Tekin S, Cummings JL. Frontal-subcortical neuronal circuits and clinical neuropsychiatry: An update. J Psychosom Res 2002;53:647-654.

20. Tullberg M, Fletcher E, DeCarli C, et al. White matter lesions impair frontal lobe function regardless of their location. Neurology 2004;63:246-253.

21. Cummings JL, Mega M, Gray K, Rosenberg-Thompson S, Carusi DA Gornbein J. The Neuropsychiatric inventory: comprehensive assessment of psychopathology in dementia. Neurology 1994;44:2308-2314.

22. Cummings JL. The Neuropsychiatric Inventory: assessing psychopahotlogy in dementia patients. Neurology 1997;48(5 Suppl 6):S10-S16.

23. Camozzato AL, Kochhann R, Simeoni C, et al. Reliability of the Brazilian Portuguese version of the Neuropsychiatric Inventory (NPI) for patients with Alzheimer's disease and their caregivers. Int Psychogeriatr 2008; 20:383-393.

24. American Psychiatric Association, Diagnostic and statistical manual of mental disorders, $4^{\text {th }}$ ed. Washington, DC: American Psychiatric Association; 2000.
25. Petersen RC. Mild cognitive impairment as a diagnostic entity. J Intern Med 2004;256:183-194

26. Davis HS, Rockwood K. Conceptualization of mild cognitive impairment: a review. Int J Geriatr Psychiatry 2004;19:313-319.

27. Hachinski VC, LLiff LD, Zilhka E, et al. Cerebral blood flow in dementia Arch Neurol 1975;32:632-637.

28. Hughes CP, Berg L, Danziger WL, Coben LA, Martin RL. A new clinical scale for the staging of dementia. Brit J Psychiatry 1982;140:566-572.

29. Fazekas F, Chawluk JB, Alavi A, Hurtig HI, Zimmerman RA. MR signal abnormalities at $1.5 \mathrm{~T}$ in Alzheimer's dementia and normal aging. AJR Am J Roentgenol 1987;149:351-356.

30. Ingles JL, Wentzel C, Fisk JD, Rockwood K. Neuropsychological predictors of incident dementia in patients with vascular cognitive impairment, without dementia. Stroke 2002:33:1999-2002.

31. Gupta M, Dasgupta A, Khwaja A, et al. The profile of behavioral and psychological symptoms in vascular cognitive impairment with and without dementia. Ann Indian Acad Neurol 2013;16:599-602.

32. Fernández-Martínez M, Castro J, Molano A, Zarranz J, Rodrigo R, Ortega R. Prevalence of neuropsychiaric symptoms in Alzheimer's disease and vascular dementia. Curr Alzheimer Res 2008:5:61-69.

33. Bandyopadhyay TK, Biswas A, Roy A, et al. Neuropsychiatric profiles in patients with Alzheimer's disease and vascular dementia. Ann Indian Acad Neurol 2014;17:325-330

34. Rockwood K, Moorhouse PK, Song X, et al. Disease progression in vascular cognitive impairment: Cognitive, functional and behavioural outcomes in the Consortium to Investigate Vascular Impairment of Cognition (CIVIC) cohort study. J Neurol Sci 2007;252:106-112.

35. Fuh JL, Wang SJ, Cummings JL. Neuropsychiatric profiles in patients with Alzheirmer's disease and vascular dementia. J Neurol Neurosurg Psychiatry 2005;76:1337-1341.

36. Frisoni B, Rozzini L, Gozetti A, et al. Behavioral syndromes in Alzheimer's disease: description and correlates. Dement and Geriatr Cogn Disord 1999:10:130-138.

37. Aalten P, Verhey FR, Bozii M, et al. Consistency of neuropsychiatric syndromes across dementias: results from the European Alzheimer Disease Consortium. Part II. Dement Geriatr Cogn Disord 2008;25:1-8.

38. Truzzi A, Ulstein I, Valente $L$, et al. Patterns of neuropsychiatric subsyndromes in Brazilian and Norwegian patients with dementia. Int Psychogeriatr 2013;25:228-235.

39. Levy R, Dubois B. Apahty and the functional anatomy of the prefrontal cortex-basal ganglia circuits. Cereb Cortex 2006;16:916-928.

40. Chin J, Seo SW, Kim SH, et al. Neurobehavioral dysfunction in patients with subcortical vascular mild cognitive impairment and subcortical vascular dementia. Clin Neuropsychol 2012;26:224-238.

41. Stella F, Forlenza OV, Laks J, et al. The Brazilian version of the neuropsychiatric inventory-clinician rating scale (NPI-C): reliability and validity in dementia. Int Psychogeriatr 2013;25:1503-1511.

42. Staekenborg SS, Su T, van Straaten ECW, et al. Behavioural and psychological symptoms in vascular dementia; differences between small- and large vessel disease. J Neurol Neurosurg Psychiatry 2010; 81:547-551.

43. Kim HJ, Kang SJ, Kim C, et al. The effects of small vessel disease and amyloid burden on neuropsychiatric symptoms: a study among patients with subcortical vascular cognitive impairments. Neurobiol Aging 2013;34:1913-1920.

44. Pan WD, Yoshida S, Liu Q, et al. Quantitative evaluation of severity of behavioral and psychological symptoms of dementia in patients with vascular dementia. Transl Neurodegener 2013;2:9.

45. Gupta M, Dasgupta A, Khwaja GA, et al. Behavioural and psychological symptoms in poststroke vascular cognitive impairment. Behav Neurol 2014;2014:430128

46. D'Onófrio G, Sancarlo D, Panza F, et al. Neuropsychiatric symptoms and functional status in Alzheimer's disease and vascular patients. Curr Alzheimer Res 2012:9:759-771. 